\title{
Multiple Sites of Associative Odor Learning as Revealed by Local Brain Microinjections of Octopamine in Honeybees
}

\author{
Martin Hammer and Randolf Menzel ${ }^{1}$ \\ Institut für Neurobiologie \\ Freie Universität Berlin \\ 14195 Berlin, Germany
}

\begin{abstract}
In a classical conditioning procedure, honeybees associate an odor with sucrose resulting in the capacity of the odor to evoke an appetitive response, the extension of the proboscis (PER). Here, we study the effects of pairing an odor with injections of octopamine (OA) as a substitute for sucrose into three putative brain sites of odor/ sucrose convergence. OA injected into the mushroom body (MB) calyces or the antennal lobe but not the lateral protocerebral lobe produces a lasting, pairing-specific enhancement of PER. During pairings, $\mathrm{OA}$ injected into the MB calyces results in an additional pairing-specific effect, because it does not lead to an acquisition but a consolidation after conditioning. These results suggest that the neuromodulator OA has the capacity of inducing associative learning in an insect brain. Moreover, they suggest the antennal lobes and the calyces as at least partially independent sites of associating odors that may contribute differently to learning and memory consolidation.
\end{abstract}

\section{Introduction}

A general question in understanding learning and memory is whether learning is induced at multiple independent sites that may contribute different aspects of what is learned and differ in the way acquired information is stored (Squire 1987; Gluck and Granger 1993). Increasing evidence suggests

${ }^{1}$ Corresponding author. that learning results in different memory phases that are processed in parallel (Gluck and Granger 1993; Folkers et al. 1993; Tully et al. 1994; Menzel and Müller 1996), and it might be that these multiple memory phases are represented at multiple sites within the brain. A related question is whether different mechanisms of cellular plasticity contribute different aspects of a learning task (Squire 1987) or whether a common mechanism is used in distributed parallel processing systems as suggested by theoretical studies (e.g., Rumelhart et al. 1986). Answering these questions requires the identification of brain and cellular sites of stimulus convergence, of cells that mediate the stimuli to be associated, and of transmitters underlying memory induction and maintenance.

In bees, restrained animals learn to associate an odorant with a sucrose reward. Three putative sites of convergent representation of information are known owing to the identification of a neuron, the VUMmx1 neuron, that mediates the reinforcing function of the unconditioned stimulus (US) in the conditioning of the proboscis extension response (PER) (Hammer 1993). Bees associate an odor [conditioned stimulus (CS)] with sucrose applied to the antennae and proboscis (US) (Bitterman et al. 1983; Menzel 1990). This results in an enhanced probability of odor-evoked PER, that lasts for hours after a single learning event and for days after only three trials (Menzel 1990). Former studies implicated the mushroom bodies (MB) in odor learning and memory in both honeybees and fruitflies. In Drosophila, flies with chemically ablated MBs or with structural MB mutations are defective in associative learning (Heisenberg et al. 1985; de Belle and Heisenberg 1994), and the gene products of the biochemical memory mutants dunce, rutabaga, and leonardo are enriched in the MBs (Davis 1993; Skoulakis and Davis 1996). In bees, local cooling of the MBs disrupts memory formation af-

LEARN IN G \& MEM ORY 5:146-156 @ 1998 by Cold Spring Harbor Laboratory Press ISSN 1072-0502/98 \$5.00

$$
\begin{array}{lllllllllllllll}
L & E & A & R & N & I & N & G & \underset{\mathbf{1 4 6}}{\boldsymbol{X}} & M & E & M & O & R & Y
\end{array}
$$


ter single-trial learning (Menzel et al. 1974; Erber et al. 1980), and several pharmacological studies have investigated global and local effects of neuroactive substances on memory storage and retrieval (Menzel et al. 1990, 1994; Erber et al. 1993). These studies show ed that $O A$, a general neuromodulator in arthropods (Kravitz 1988; Bicker and Menzel 1989), facilitates memory storage and retrieval when injected into the MBs and rescues conditioning selectively when injected into reserpine treated animals, whereas serotonin inhibits conditioning and memory retrieval, and dopamine appears to be selectively involved in the motor components of learned responses (R. Menzel, A. Hayne, and C. Kinzel, in prep.).

Activity of the VUMmx1 neuron substitutes for the reinforcing function of the US (sucrose): Stimulating the neuron intracellularly supports conditioned odorevoked proboscis extension when stimulation of the neuron is paired with an odorant (Hammer 1993). This neuron innervates three neuropils involved in odor (CS) processing: the antennal lobe glomeruli, the primary olfactory neuropil in insects; the MB calyces, which receive input from the antennal lobe; and the lateral protocerebral lobe, which presumably provides descending brain output. The lateral protocerebral lobe receives both direct and MB-processed input from the antennal lobe. Moreover, VUMmx1 belongs to a group of octopamine (OA)-immunoreactive neurons (Kreissl et al. 1994). Therefore, OA may be released by VUMmx1, and this could mediate the US in PER conditioning. It is not yet known which of the brain areas innervated by VUMmx 1 support associative learning: Different sites may independently support learning, one site only may be sufficient (e.g., the MB calyces), or the concerted action of more than one site may be necessary.

Therefore, in a conditioning analog, the effects of pairing odor (CS) with OA injections into these brain sites, as a substitute for the US, w ere tested in otherwise intact bees. The data presented here confirm the role of $M B$ in associative learning. However, they also provide evidence that the antennal lobe acts as an additional site of associative learning in bees. Because $O A$ injections into the $M B$ calyces result in different pairing-specific effects during acquisition and memory consolidation, an effect not found for the antennal lobe, the antennal lobes and the calyces presumably contribute different aspects of learning. Finally no nonassociative effects of $O A$ were found, although both sucrose stimuli and OA affect nonassociative modi- fications of PER (Menzel et al. 1990, 1991; Braun and Bicker 1992; Hammer et al. 1994). This paper, therefore, presents evidence for a role of $O A$ in reinforcement processing during olfactory conditioning.

Some of the results have been published in abstract form (M. Hammer, R. Menzel, and U. Schneider, unpubl.).

\section{Materials and Methods}

Foraging honeybees were caught the afternoon before the experiments, harnessed in small metal tubes, fed to satiation, and kept overnight in the dark and at room temperature. Before experiments, the head capsule was opened above the site of injection, and trachea and glandular tissue was gently placed to the side to expose the brain. Bees were injected into one brain side only. To avoid direct odor-evoked activity in the other side, the antenna contralateral to the injected side was cut off. Then, bees were trained in a conditioning ana$\log$ in which eight puffs of odor stimuli (duration, $\sim 3 \mathrm{sec}$ ) separated by 3 min were either paired (interval, 2 sec after onset of odor stimulus) or unpaired (interval, $1.5 \mathrm{~min}$ ) with injections of bee saline (135 mM NaCl, $5 \mathrm{~mm} \mathrm{KCL}, 10 \mathrm{~mm} \mathrm{MgCl} 2,1.6$ $\mathrm{mm} \mathrm{CaCl} 2$ at $\mathrm{pH} 7.3)$ or $\mathrm{OA}\left(10^{-6} \mathrm{M}\right.$, in saline, $\mathrm{pH}$ 7.3). In the unpaired groups, the alternation of odor stimulation and injection started in half of the animals with the odor presentation; in the other half, with injection. Solutions were injected with pressure pulses (duration, $1 \mathrm{sec}$ ). Odors were presented by gently pushing air through a syringe that contained a piece of tissue paper soaked with carnation oil, adding a weak mechanical component to the odor stimulus. Odors, how ever, have a substantially higher salience than mechanical stimulation (Menzel 1990), and therefore, conditioned PER can be viewed as being predominantly odor evoked. The effect of treatment was measured as the probability of PER to the odor during treatment (acquisition function) and in three extinction tests with odor presentation alone $(30,40$, and $50 \mathrm{~min}$ afterwards). A response was counted only if the proboscis was fully extended and extension occurred shortly after stimulus onset. After the last test, the antennae were stimulated with sucrose to test whether the animal displayed the unconditioned response. Only animals showing the unconditioned response were included in the data analysis. Less then $10 \%$ of the animals had to be discarded because of this criterion.

$$
\begin{array}{llllllllllllllll}
\hline & E & A & R & N & I & N & G & \underset{147}{\mathbf{X}} & M & E & M & O & R & Y
\end{array}
$$


Injections were made directly into the antennal lobe, lateral protocerebral lobe, the optic lobe (as a control neuropil), or directly between the median and lateral calyx of the MBs. Injected volumes were $1 \mathrm{nl}$ per injection for the MB calyces, the optic lobes, and the lateral lobe and $0.6 \mathrm{nl}$ for the antennal lobe to adjust for its smaller volume. Volumes were calibrated by injecting solutions into a small vessel containing paraffin oil both before the first injection and whenever the microcapillary was used again. Volumes in the range of 0.5-1 $\mathrm{nl}$ injected into one MB or one antennal lobe are known to need $>5$ min to exert an action on the respective contralateral side (Menzel et al. 1988; U. Müller, pers. comm.). Further evidence for the local action of OA comes from results presented here that $O A$ injected into the lateral protocerebrum has no effect, indicating that it also does not diffuse to the MB and antennal lobe sufficiently enough to induce the respective effect there (see below). To obtain an estimate of the success and the restriction of the injected solutions to the respective neuropils, Lucifer Yellow (LY, $0.1 \%)$ was added. Immediately after experiments, bees were injected with $4 \%$ paraformaldehyde. Brains were dissected under saline, dehydrated, cleared in methylsalicylate, and examined under a fluorescence microscope for the distribution and localization of LY. Assessment of the distribution of LY was carried out without knowledge of the experimental history of animals. All animals of the experimental groups show ed $L Y$ to be restricted to the respectively injected neuropils. Animals with diffuse LY distribution were discarded, and animals in which injection was unsuccessful were taken as an additional control group ("missed injection") because they were treated like the experimental groups but did not receive a successful injection. The proportion of animals discarded because of diffuse injection ranged form $10 \%-20 \%$.

Statistical analyses of data was carried out with Statsoft Statistica. Within-group comparison of response frequencies betw een the last three trials of acquisition and tests used the Cochrans $\mathrm{Q}$ test. Betw een-group comparison of acquisition (OA vs. saline) used the Mann-Whitney U test by evaluating the total number of PERs during acquisition for each individual. A log-linear analysis on the frequencies of responding bees $w$ as used to investigate the development of odor-evoked PER (\%PER) during repeated tests (multiple extinction tests). Interactions of the design variables (successive tests, odor and injections paired or unpaired, in- jected solutions) with the development of the response frequencies were considered to be significant, if both partial $\left(\chi_{p}{ }^{2}\right)$ and marginal $\left(\chi_{m}{ }^{2}\right)$ associations led to a probability level of $\mathrm{P}<0.05$. In Results only the $\chi_{p}{ }^{2}$ values are presented.

\section{Results}

ODOR PAIRED WITH LOCALLY INJECTED OA PRODUCES PAIRING AND SITE-SPECIFIC ENHANCEMENT OF PER

To investigate whether the putative transmitter of the VUMmx 1 neuron, OA, substitutes for the US in olfactory PER conditioning, bees were trained in a conditioning analog in which the US was replaced by $O A$ injections into those brain structures that are innervated by VUMmx1, the MB calyces, the antennal lobes, and the lateral protocerebral lobe (Fig. 1C). If one of these structures is sufficient to produce conditioning, pairing an odor with locally injected OA should enhance the odorevoked probability of PER. The distribution of injected solutions was monitored by LY (Fig. 1A,B). In the case of the MB calyces and the antennal lobe, LY was restricted to the injected structure. Bees were discarded when LY diffused beyond the calyces or antennal lobe (see Materials and Methods). When solutions were injected into the lateral protocerebral lobe or the optic lobes, LY was less restricted, presumably because these neuropils are less compartmentalized. Therefore, only those bees were analyzed in which LY had not diffused into the MB calyces or the antennal lobes. In a number of bees, the injection was-for technical reasons- unsuccessful. This allowed us to include an additional control group that was run through the whole experiment without receiving an injection [missed injection (mInj)]. Bees that did not respond to antennal sucrose stimulation after the tests or died during the experiment (together 13\%$18 \%$, effects not treatment specific) were discarded. During the conditioning procedure, bees occasionally (4\%) extended their proboscis independently of stimulation or injection. These bees were included in the respective groups, and only their response following odor stimulation was evaluated. Because there was no difference between groups, these effects were not treatment specific. The conditioning procedure also allowed us to test whether the injection of $O A$ led to any changes in behavior (e.g., movements of antennae or mouthparts) or in the probability of spontane-

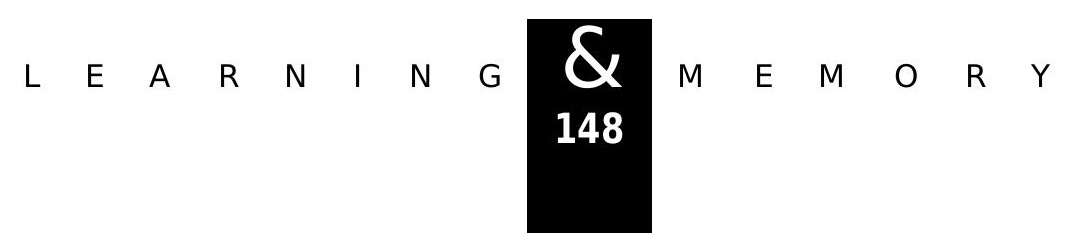



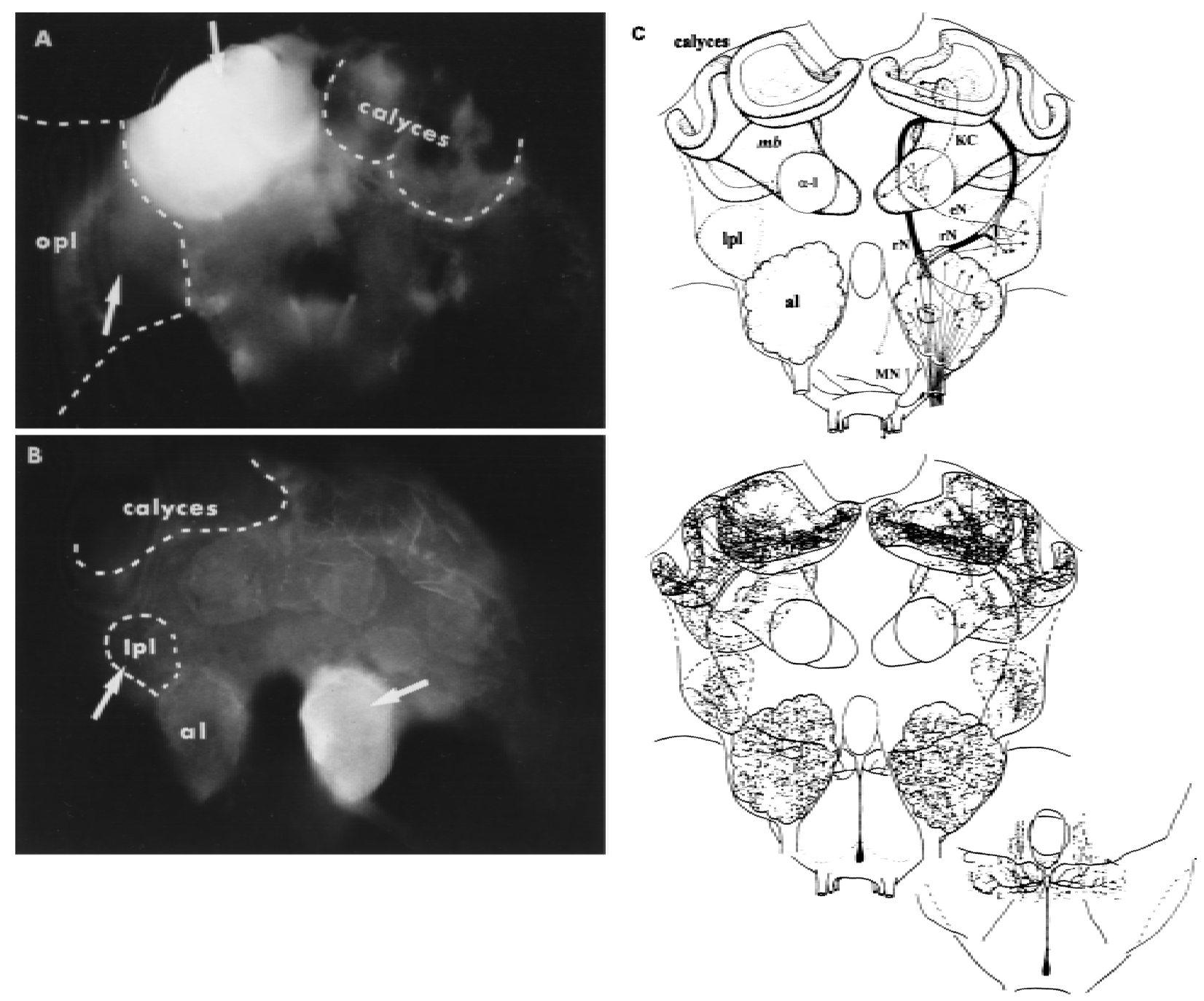

Figure 1: Distribution of LY injected together with test solutions in two brain sites innervated by the VUM mx1 neuron. (A) Distribution in the MB calyces; back view of the brain. (B) Distribution in the antennal lobe (al); frontal view. (C) Schematic diagram of the olfactory (CS) pathway; frontal view (top) and dendritic arborizations of the VU M mxI (bottom) (modified after Hammer 1993). ( $\alpha$-1) $\alpha$-Lobe, one of the MB-output lobes; (Ipl) lateral protocerebral lobe; (opl) optic lobes; (rN) two tracts of relay neurons connecting al with MB calyces and Ipl; (KC) Kenyon cells; (eN) M B-extrinsic neurons connecting $\alpha$-I with Ipl; (MN) motor neurons of the proboscis; (mb) mushroom body. Arrows (in $A, B$ ) indicate the site of injection.

ous responding to the $\mathrm{CS}$. This is not the case: On average, $10 \%$ of the bees extended the proboscis to the CS before conditioning, and this proportion did not change with injection excluding the possibility of unspecific arousal or sensitization effects.

When solutions were injected into the MB calyces (Fig. 2), \%PER during the three tests revealed a pairing-specific effect of $\mathrm{OA}$. A log-linear analysis yielded a significant interaction between injected solutions (i.e., $O A$ vs. saline ) and \%PER $\left(\chi_{p}^{2}=5.584, \mathrm{P}<0.018 ; \mathrm{df}=1\right.$ each $)$ and between the temporal relation between odor stimuli and injection (i.e., paired vs. unpaired ) and \%PER $\left(\chi_{\mathrm{p}}{ }^{2}=4.926, \mathrm{P}<0.027 ; \mathrm{P}<0.031 ; \mathrm{df}=1\right)$ but not between successive tests and \%PER. The effect is pairing specific because the response level of the OA unpaired group did not differ from that of saline controls. As revealed by LY, injection was unsuccessful in 12 bees (group: mlnj). Because they rarely responded to the odor, this additional control group shows that calyx injections themselves affects PER (not injected bees compared with saline unpaired, $\chi_{\mathrm{p}}{ }^{2}=7.5, \mathrm{P}<0.01$, to saline paired, $x_{\mathrm{p}}^{2}=8.5, \mathrm{P}<0.005$, and to OA unpaired, 


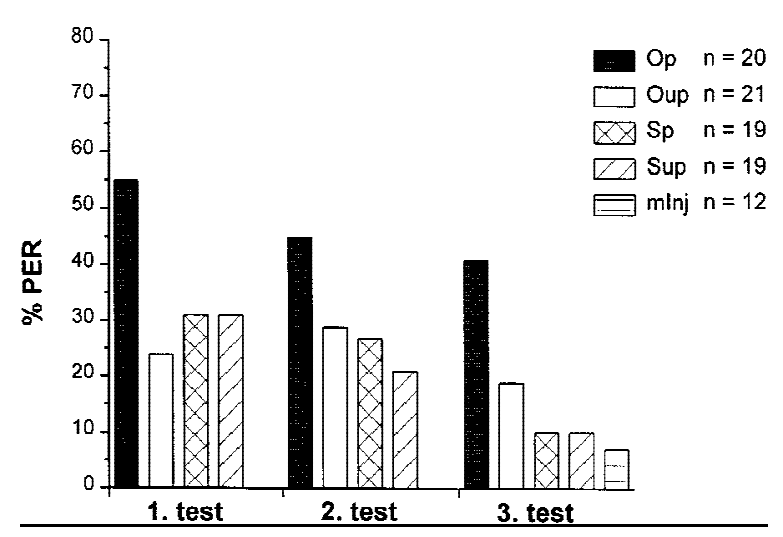

Figure 2: Effects of odor (CS) paired with local injections of $O A$ (US) into the MB calyces on \%PER during three consecutive test trials 30,40 , and $50 \mathrm{~min}$ after injection. Odor either paired with OA $(0 p)$ or bee-saline (Sp) or unpaired with OA (O up) or saline (Sup) injected into the MB calyces. A sham control group were animals in which injection was unsuccessful (mlnj). Spontaneous response level to the CS before conditioning was $\sim 10 \%$. The $O A$ paired group $(O p)$ shows an increased level of \%PER. ( $n$ ) The number of subjects per group that survived throughout experiments, showed LY restricted to the injected neuropil, and gave a sucroseevoked PER after the last test. The mInj group gave zero response at test 1 and 2 .

$\left.\chi_{p}^{2}=9.298, P<0.005 ; d f=1\right)$. This injection effect is not pairing specific and does not differ from the unpaired injection of OA. It can be concluded, therefore, that the injection of saline leads to some unspecific enhancement of responsiveness to an odor stimulus possibly owing to an ionic or osmotic mismatch between saline and extracellular fluid.
To investigate whether the effect of $O A$ is restricted to injections into the calyces, OA or saline was injected each into the antennal lobe. To avoid problems of interpretation in case of negative results, two additional groups with $O A$ injected into MB calyces either paired or unpaired with odor were run in parallel. The antennal lobe-injected bees showed a pairing-specific effect of OA (Fig. 3). The interaction between injected solutions and $\%$ PER $\left(\chi_{p}^{2}=12.75, \quad P<0.0005 ; \mathrm{df}=1\right)$ and between paired versus unpaired and \%PER $\left(\chi_{\mathrm{p}}{ }^{2}=5.667, \mathrm{P}<0.02 ; \mathrm{df}=1\right)$ was significant, but not betw een successive tests and \%PER. In contrast to the first experiment, however, there was no unspecific effect of the injection itself as revealed by comparing the $\mathrm{mlnj}$ bees $(n=29)$ with the three controls. The two groups that were injected into the MB calyces confirmed the results of the first experiment with calyces injections. The somewhat higher response level during the tests $(\sim 67 \%$ compared with $47 \%$ for the paired group, averages across the tests in the two experiments) was pairing specific $\left(\chi_{p}^{2}=11.69, \mathrm{P}<0.001 ; \mathrm{df}=1\right)$.

To investigate the role of the lateral protocerebral lobe, odor stimuli were paired with $O A$ injections into the MB calyces, the lateral protocerebral lobe that adjoins the antennal lobe, and the optic lobes that are not innervated by VUMmx1 (Fig. $1 B, C)$. From 20 bees that were not injected, two responded occasionally. Only bees that received injections into the MB calyces showed a significantly increased \%PER during tests (Fig. 4) (comparison between MB-calyx injection and lateral lobe injection: $x_{p}{ }^{2}=20.923, P<0.0001 ; d f=1$ ).

Taken collectively, these results suggest that
Figure 3: Effects of odor (CS) paired with local injections of OA (US) into MB calyces or antennal lobe on \%PER during three consecutive test trials 30,40 , and 50 min after injection. 0 dor paired with OA (OpA) or saline (SpA) or unpaired with O A (O upA) or saline (SupA) injected into the antennal lobe and an unsuccessfully injected control group (mlnj), and two parallel groups with odor paired $(\mathrm{O} P \mathrm{C})$ or unpaired (OupC) with $\mathrm{OA}$ injected into the MB calyces. The OA paired groups show an increased level of $\%$ PER. (n) The number of subjects per group that survived throughout experiments, showed LY restricted to the injected neuropil, and gave a sucroseevoked PER after the last test.

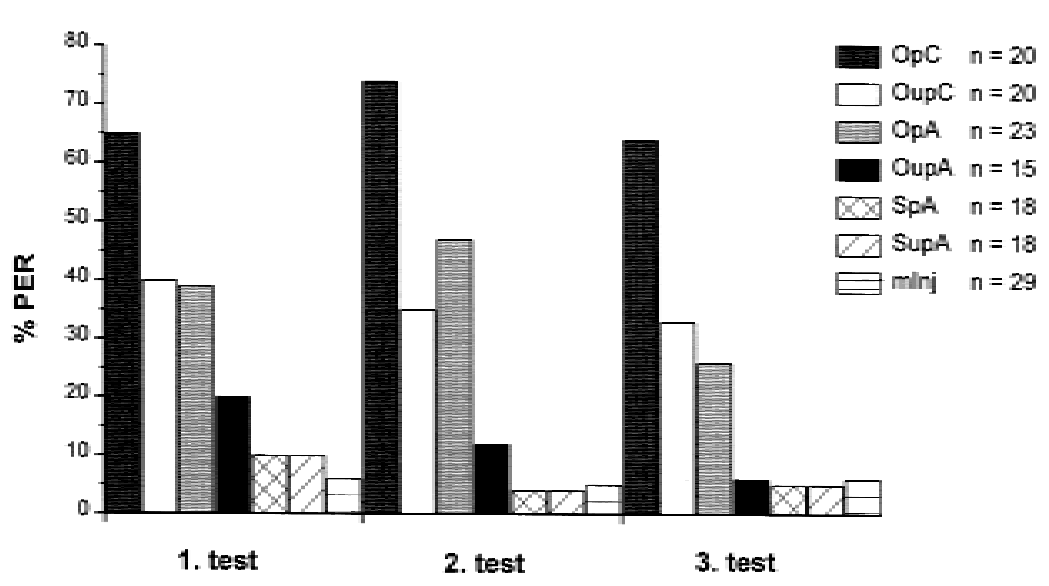




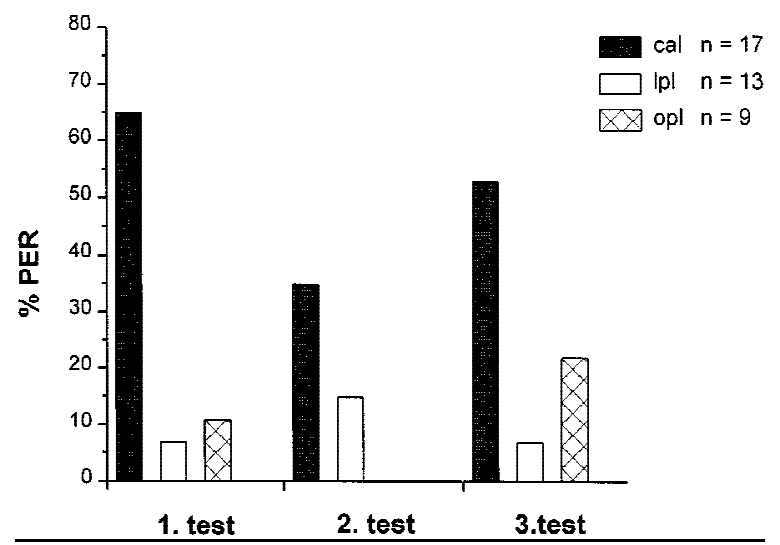

Figure 4: Effects of odor (CS) paired with local injection of OA (US) into M B calyces (cal), lateral protocerebral lobe (Ipl), or optic lobe (opl) on \% PER during three consecutive test trials 30,40 , and 50 min after injection. The cal group shows an increased level of \% PER. (n) The number of animals per group that survived throughout the experiment, showed LY restricted to the injected neuropil, and gave a sucrose-evoked PER after the last test.

odorevoked neural activity and OA injections provide a pairing-specific mechanism to associate an odor. Surprisingly, this holds true for both the antennal lobes and the MB calyces. Both appear to be sites of associative learning. Each site appears not to require $O A$ release in the other. It is not known, however, whether the odor/OA injection pairing effect would lead to stronger learning if $O A$ w ould be injected simultaneously in the calyx and antennal lobe. It is also not know $n$ whether the antennal lobes could support the pairing-specific effect if the MB would be ablated. Both kinds of experiments turned out to be technically impossible so far. The lateral protocerebral lobe, at least, is either not directly involved in OA-mediated learning or, because it lies downstream to the antennal lobe and the MBs, might require associative processes in at least one of these neuropils. Furthermore, a lack of an OA effect for injections into the optic lobes additionally argues for site specificity of the injections.

\section{ACQUISITION FUNCTIONS REVEAL A SECOND OA} PAIRING AND SITE-SPECIFIC EFFECT

The procedure applied in PER conditioning allows for an evaluation of the acquisition process, because the CS (odorant) is given $2 \mathrm{sec}$ before the onset of the US (here, injection of OA in the paired groups). In usual PER conditioning with multiple- trial CS/US (sucrose) pairings, conditioning yields a rapid acquisition that reaches its asymptotic level within a few trials (Bitterman et al. 1983; Menzel and Müller 1996). To investigate whether OA also mimics the acquisition process during multipletrial conditioning, acquisition functions of odorevoked PER were investigated for MB calyx and antennal lobe injections. Again, paired and unpaired groups both for $O A$ and saline were compared, and the \%PER during the 2 sec following odor stimulation was evaluated. Figure 5 show s the results for MB calyx-injected bees (same experiment as Fig. 2, groups Op, Oup, Sp, and Sup) and for antennal lobe-injected bees (same experiment as Fig. 3, groups OpA, OupA, SpA, and SupA).

Surprisingly, for the MB-calyces injections, acquisition in the odor/OA paired yielded low acquisition and a significantly lower level of odorevoked PER at the acquisition asymptote as compared with that in the three extinction tests (Q test: $\mathrm{OA}$ paired $\mathrm{Q}=33.95, \mathrm{df}=0.0001$ ). All three other groups (saline paired, saline unpaired, and OA unpaired) show an acquisition process and no difference between the end of acquisition and the extinction trials [OA unpaired $\mathrm{Q}=3.06$, saline paired $\mathrm{Q}=4.12$, saline unpaired $\mathrm{Q}=8.79$, not significant (NS) for each case] (Fig. 5, calyx). A comparison with the saline paired group indicated that the reduced responsiveness during acquisition is OA specific (OA paired against saline paired, $U$ test: $\mathrm{z}=2.124, \mathrm{P}<0.05)$. The acquisition functions for the two unpaired controls (OA and saline) did not differ $(z=0.624, N S)$. An additional experiment (data not shown) with a slightly different stimulus protocol confirmed these results. In this experiment, an odor was delivered in three consecutive sessions, either paired with OA or saline; each session consisted of three presentations (1.5-min interval) each, separated by $5 \mathrm{~min}$. The \%PER during extinction tests (OA $\sim 35 \%$, saline $22 \%$ ) depended on the injected solution $\left(\chi_{\mathrm{p}}{ }^{2}=6.034, \chi_{\mathrm{m}}{ }^{2}=5.98\right.$, $\mathrm{P}<0.014 ; \mathrm{df}=1$; OA $\mathrm{n}=43$, saline $\mathrm{n}=43$ ). There was an increase between the asymptotic level of $P E R$ and the three later tests for $O A(Q=61.72$, $\mathrm{df}=5 \mathrm{P}<0.0001)$ but not saline $(\mathrm{Q}=9.66, \mathrm{df}=5$, $\mathrm{P}<0.09)$, and a group-by-group comparison yielded a difference of the acquisition functions dependent on the solution produced by the low level of responding bees injected with $O A$ (U test: $\mathrm{z}=1.962, \mathrm{P}<0.05)$. Both these experiments indicate that pairing an odor and $O A$ injected into the $M B$ calyces doe not mimic the steep acquisition observed in PER conditioning. Apparently, there is 
Hammer and Menzel

Figure 5: Pairing-specific effects of $O A$ injected into the MB calyces or the antennal lobes on acquisition of PER and during tests (same experiments as Fig. 3). O dor paired or unpaired with OA (O) or saline $(O)$ injected into the MB calyces, and odor paired or unpaired with $O A(O)$ or saline $(O)$ injected into the antennal lobe. Between-group comparison of the acquisition function reveals a significant reduction of the \%PER, and within-group comparison reveals a significant increase in the $\% \mathrm{PER}$ in the group that received pairings of odor/O A MB-calyces injections.
Calyx

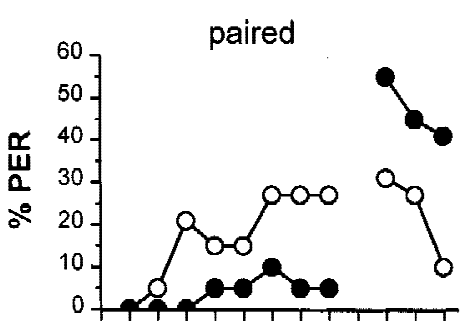

Antennal Lobe
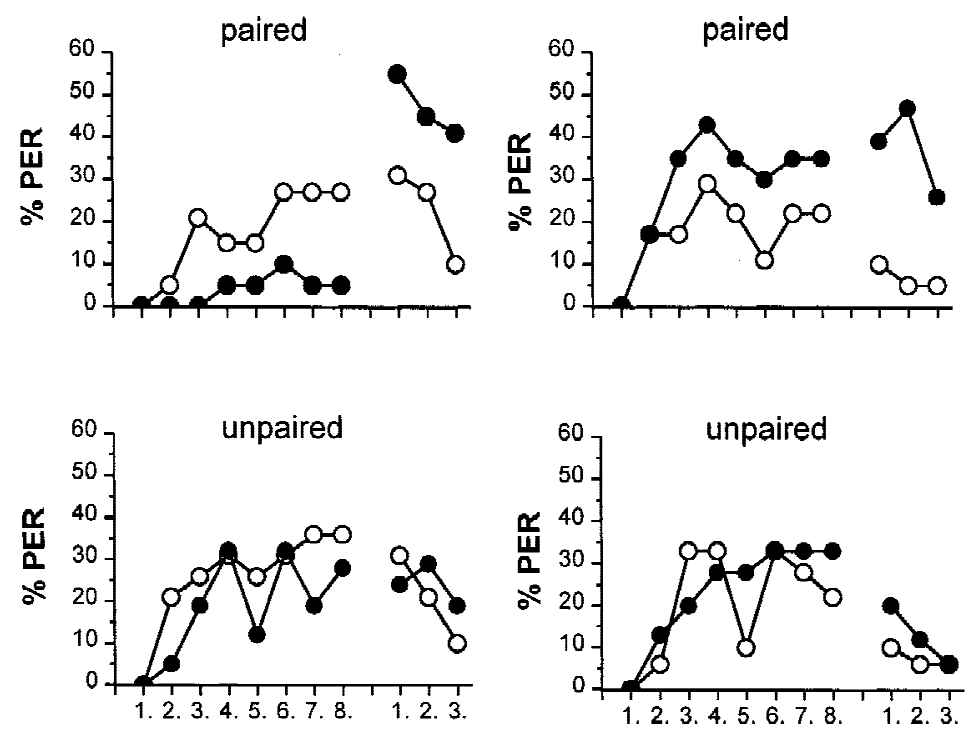

acquisition trial test

acquisition trial test also no first-trial effect, a phenomenon w ell know $n$ from usual PER conditioning, because in the OA paired groups no bee responded during the second trial. Rather, OA appears to be of little effect during acquisition (with even some indication, though not statistically significant, of an inhibitory effect when compared with the control group) but induces pairing specific memory consolidation over the period of 30 min following conditioning.

In the antennal lobe-injected groups (Fig. 5, antennal lobe), the acquisition process leads to a rising \%PER function that is significantly higher at the asymptotic level for odor/OA pairing as compared with odor/OA pairing in the calyx. Conditioned PER level at the first extinction trial is either not different from the asymptotic level of acquisition (OA paired group and OA unpaired group: NS, $\mathrm{Q}=9.89, \mathrm{P}<0.08$ ) or is lower ( $\mathrm{OA}$ unpaired group and the two saline groups: saline paired, $\mathrm{Q}=11.67, \mathrm{P}<0.05$; saline unpaired, $\mathrm{Q}=15.2$, $\mathrm{P}<0.01)$. Thus, injecting solutions into the antennal lobe produced an enhancement of odor-evoked PERs during acquisition. This enhancement, however, is maintained over a longer period only for bees that received odor/OA pairings. Antennal lobes and MB calyces appear to differ in a very important property with respect to $O A$ pairing with CS. Antennal lobes when OA injected support an acquisition process but no memory consolidation; MB when OA injected do not show an acqui- sition process but do show memory consolidation. This effect may be understood as indicating an inhibitory phenomenon at the level of MBs during acquisition or a memory consolidation process induced by the MBs independent of acquisition. If, how ever, the MBs participate in fast memory processing in vivo, other transmitters activated by the US or OA released in more than one neuropil should be necessary. For instance, acquisition may require associative learning in the antennal lobe that also facilitates memory formation in the MBs.

\section{Discussion}

Injections of the general neuromodulator $\mathrm{OA}$ can act as a substitute for the reinforcing function of sucrose US in PER conditioning. Because OA is the putative transmitter of the VUMmxl neuron (Kreissl et al. 1994) whose activity can also substitute for the US reinforcing function (Hammer 1993), this suggests that CSevoked neural activity and $O A$ released from $V U M m x 1$ initiate pairingspecific mechanisms to associate a CS with sucrose. Moreover, the finding that $\mathrm{OA}$ injection into antennal lobe and MB calyces induces independently olfactory memories, that differ with respect to acquisition and consolidation, supports the notion that memory formation occurs at multiple sites in parallel and with functionally distinct aspects (Menzel and Müller 1996). If the distribution 
of $L Y$ injected together with $O A$ is considered to be indicative for the localization of $\mathrm{OA}$ action, it can be concluded that neither the lateral protocerebrum nor the optic ganglia are involved in these functions but only the MB calyces and the antennal lobe, though with differential mechanisms.

Two observations support this interpretation. (1) LY did not diffuse from one to the other site; (2) if $L Y$ would diffuse less readily than $O A$ one should find a conditioning effect when injected into the lateral protocerebrum, because this structure is closer to the MB calyces and is less well-ensheathed by glia than the antennal lobes and the calyces. This was not found. These results do not rule out the possibility of more effective learning if both symmetrical structures (the two antennal lobes and the two MBs) or the two structures on each side (ipsilateral antennal lobe and $M B$ ) are provided with $O A$ simultaneously as is the case when excitation in VUMmxI leads to OA release.

That the antennal lobe (besides the MB) is involved in learning was indicated already by the finding that cooling of the antennal lobe immediately after single-trial conditioning induces amnesia (Menzel et al. 1974; Erber et al. 1980). Further evidence comes from studies that document learningdependent changes in the antennal lobe ( $T$. Faber, J. Joerges, and R. Menzel, unpubl.) and computational models that attempt to simulate behavioral learning phenomena with the properties of the circuitry of the antennal lobe (Malaka and Hamer 1996). The results presented here emphasize that the antennal lobes directly contribute to associative learning. It is tempting to speculate that the antennal lobes may perform some basic form of odor learning possibly related to odor discrimination and odor evaluation, whereas the MBs with their multimodal inputs (Mobbs 1982, 1984) may participate in contextual and multisensory learning (Rybak and Menzel, this issue).

Although several studies implicate the role of MBs in olfactory learning and memory (Heisenberg et al. 1985; Davis 1993; de Belle and Heisenberg 1994), it is not yet know $n$ whether learning-related neural plasticities are localized at the MB inputs, the calyces, or their outputs, the lobes, or at both sites. In bees, the time course of developing resistance to retrograde amnesia produced by local cooling of the calyces closely resembles that of cooling the whole animal, whereas that induced by cooling the $\alpha$-lobe is faster (Erber et al. 1980), indicating that associative memory formation is more closely related to the calyces (Hamer and Menzel
1995). Consistent with this view, the present study confirms that pairings of $\mathrm{CS} / \mathrm{OA}$ injections into the calyces are sufficient to initiate associative memory.

However, OA injection into the calyces does not mimic PER conditioning completely. It fails to produce an acquisition-like increase in the odorevoked probability of PER. Rather, the effect of odor and OA-calyx injection pairings needs time to develop, whereas odor and OA-antennal lobe injection pairings lead to an acquisition-like function with a stable but not improved forward pairing memory effect as compared with the OA-calyx injection pairings. The low acquisition of the group receiving forward odor and OA-calyx injection pairings may either be interpreted as being indicative for a consolidation process establishing a memory trace elsew here under the guidance of the MB calyces or as an inhibitory phenomenon, which lasts throughout the multiple pairing trials but disappears after a rest interval of $\geqslant 30 \mathrm{~min}$. After this interval, the pairing effect is selective both for $\mathrm{OA}$ (as compare with saline) and to the forward sequence of odor and injection (as compared with the unpaired sequence; Fig. 5, calyx). Inhibitory phenomena are known from OA injection into the bee brain. Erber and Kloppenburg (1995) found that OA injected into optic lobes in the same concentration as used here results in a transient reduction follow ed by a lasting increase of a visual direction sensitivity.

Previous studies showed that $O A$ facilitates memory storage and retrieval in bees (Menzel et al. 1990). These findings are compatible with a general modulatory function of $O A$. The relationship between a general modulatory or arousing transmitter and a transmitter signaling reinforcement has yet to be analyzed (Hammer and Menzel 1994). In mollusks, for example, evidence favors the interpretation that both functions are closely related or identical. In Hermissenda (Hodgson and Crow 1992) and Aplysia (Byrne 1987), the neuromodulator serotonin induces both nonassociative and associative behavioral and cellular modifications. In the mammalian brain, several modulatory systems based on different primary transmitters act in parallel both at the circuit and the cellular levels. Dopamine appears to mediate appetitive reinforcement at least in the context of motor learning via pathways originating in the ventral striatum (Mirenowicz and Schultz 1996; Schultz et al. 1997). In insects, both $O A$ and dopamine have been related to the reinforcing function of brain circuits (bee:

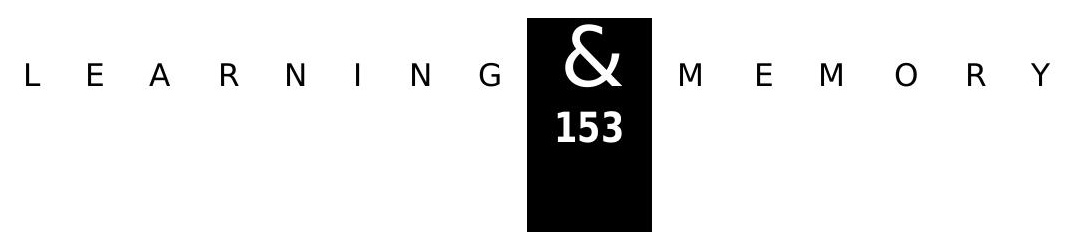


Menzel and Müller 1996; Drosophila: Davis 1996), but the separation between general modulatory and specific reinforcing actions are only beginning to be understood for $\mathrm{OA}$ in the bee brain. Recent data indicate that $O A$ is necessary for olfactory conditioning, because it repairs specifically conditioning in reserpine-treated bees ( $R$. Menzel, A. Heyne, C. Kinzel, A. Fiala, and B. Gerber, in prep.). That study and the findings reported here together with the observation that $\mathrm{OA}$ injections did not enhance arousal or sensitization as measured by the response probability to an odor stimulus without conditioning suggest a more specific role of $O A$, namely, its function as a primary messenger of the US pathway with its reinforcing function.

However, OA is probably not the reinforcement mediating transmitter for all forms of learning in bees. Visual ganglia and the visual part of the $M B$, the collar of the calyx, are not innervated by VUM neurons, and whether OA immunoreactive neurons innervate visual ganglia and the visual part of the MBs, the collar, is yet unclear (Kreissl et al. 1994). Thus, additional transmitters are likely to exist that serve the function of reinforcement for other forms of learning than olfactory conditioning in bees.

Primary transmitters signaling reinforcement to target neurons induce cellular signaling cascades leading to learning-specific changes. As suggested for Drosophila and Aplysia (Byrne 1987; Byrne et al. 1993; Davis 1993), also in bees, the induction of associative learning could involve the CAMP signal cascade, because in the antennal lobe OA, cAMP and sucrose stimulate a CAMP-dependent protein kinase A (Hildebrandt and Müller 1995a,b). Receptor binding studies show that the MBs in bees may express specific OA receptors (Erber et al. 1993). That the OA concentration used here stimulates other aminergic systems, such as serotonin, that have antagonistic effects to $O A$ seems unlikely, because $1 \mu \mathrm{m}$ serotonin but not $1 \mu \mathrm{M} O \mathrm{~A}$ inhibits the binding of $\left[{ }^{3} \mathrm{H}\right] \mathrm{LSD}$ to brain serotonin receptors (Blenau et al. 1995). Independent of their nature, second-messenger effects that underlie associative learning must depend on the convergence and coincident action of the CS and the US pathway. Several candidate neural populations exist for this convergence. In, for example, the calyces, Kenyon cells could act as postsynaptic convergence sites or presynaptic convergence could occur at olfactory relay neurons, suggesting an activity-dependent form of neuromodulation for OA. Alternatively, simultaneous activity of relay neurons and Kenyon cells could mediate a Hebbian form of learning. In locusts, relay neurons drive synchronous membrane potential oscillation in the olfactory pathway (Laurent and Davidowitz 1994; Laurent and Naraghi 1994), and oscillations of local field potentials in honeybees were suggested to contribute to olfactory stimulus generalization (Stopfer et al. 1997). Synchronization of intracellular activity and field potentials could facilitate Hebbian plasticity as suggested for the piriform cortex (Hasselmo et al. 1990). In bees, OA-dependent neuromodulation would then have a permissive pairing-specific function in the induction of Hebbian plasticity.

Irrespective of what the cellular mechanisms of pairing are, our study shows that associative olfactory memory in the bee brain is established at two sites independently of each other. These two sites apparently differ in their properties as they relate to acquisition and consolidation. The concept of temporal memory phases is w ell supported in human and animal memory research, but the relationship between temporal sequences of memory formation and their respective localization within the brain is very hard to tackle, and only the hippocampus may have been analyzed in this respect sufficiently well so far (Squire 1987). The approach applied here offers the opportunity to explore the contributions of two intimately connected centers of neural integration, that of primary sensory integration (antennal lobe) and of higher order multisensory integration (MB). The test of more complex forms of learning (e.g., context-dependent learning, second-order conditioning, sensory preconditioning, and different schedules of acquisition) will allow us to address the questions of whether these neural centers need to interact during acquisition and whether particular memory components are stored preferentially or exclusively in any one of these neuropils. These studies will shed light on the general problems of why memories appear distributed rather than localized and why they run through phases toward their final form.

\section{Acknowledgments}

We thank $U$. Schneider for her help with the experiments and Dr. U. Müller and B. Gerber for their helpful comments on earlier versions of the manuscript. This work was supported by Deutsche Forschungsgemeinschaft "Forschergruppen" grant Pf 128/6-3 (to R.M. and M.H.).

The publication costs of this article were defrayed in part by payment of page charges. This article must therefore

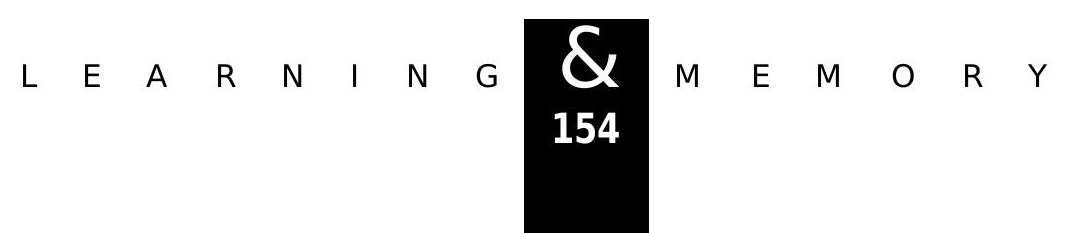


be hereby marked "advertisement" in accordance with 18 USC section 1734 solely to indicate this fact.

\section{References}

Bicker, G. and R. Menzel. 1989. Chemical codes for the control of behaviour in arthropods. Nature 337: 33-39.

Bitterman, M.E., R. Menzel, A. Fietz, and S. Schäfer. 1983. Classical conditioning of proboscis extension in honeybees (Apis mellifera). J. Comp. Psychol. 97: 107-119.

Blenau, W., T. May, and J. Erber. 1995. Characterization of $\left[{ }^{3} \mathrm{H}\right] \mathrm{LSD}$ binding to a serotonin-sensitive site in honeybee (Apis mellifera) brain. Comp. Biochem. Physiol. B 112: 377-384.

Braun, G. and G. Bicker. 1992. Habituation of an appetitive reflex in the honeybee. J. Neurophysiol. 67: 588-598.

Byrne, J.H. 1987. Cellular analysis of associative learning. Physiol. Rev. 67: 329-439.

Byrne, J.H., R. Zwartjes, R. Homayouni, S.D. Critz, and A. Eskin. 1993. Roles of second messenger pathways in neuronal plasticity and in learning and memory. Insights gained from Aplysia. In Advances in second messenger and phosphoprotein research (ed. S. Shenolikar and A.C. Nairn), vol 27, pp. 47-108. Raven Press, New York, NY.

Davis, R.L. 1993. Mushroom bodies and Drosophila learning. Neuron 11: 1-14.

- - . 1996. Physiology and biochemistry of Drosophila learning mutants. Physiol. Rev. 72: 299-317.

de Belle, J.S. and M. Heisenberg. 1994. Associative odor learning in Drosophila abolished by chemical ablation of mushroom bodies. Science 263: 692-695.

Erber, J. and P. Kloppenburg. 1995. The modulatory effects of serotonin and octopamine in the visual system of the honey bee (Apis mellifera L.) I. Behavioral analysis of the motion-sensitive antennal reflex. J. Comp. Physiol. A 176: 111-118.

Erber, J., T. Masuhr, and R. Menzel. 1980. Localization of short-term memory in the brain of the bee, Apis mellifera. Physiol. Entomol. 5: 343-358.

Erber, J., P. Kloppenburg, and A. Scheidler. 1993. Neuromodulation by serotonin and octopamine in the honeybee: Behaviour, neuroanatomy and electrophysiology. Experientia 49: 1073-1083.

Evans, P.D. 1985. O ctopamine. In Comprehensive insect physiology, biochemistry and pharmacology (ed. G.A. Kerkut and L.I. Gilbert), pp. 499-530. Pergamon, Oxford, UK.

Folkers, E., P.F. Drain, and W.G. Q uinn. 1993. Radish, a Drosophila mutant deficient in consolidated memory. Proc. Natl. Acad. Sci. 90: 8123-8127.

Gluck, M.A. and R. Granger. 1993. Computational models of the neural basis of learning and memory. Annu. Rev. Neurosci. 16: 667-706.

Hammer, M. 1993. An identified neuron mediates the unconditioned stimulus in associative olfactory learning in honeybees. Nature 366: 59-63.

Hammer, M. and R. Menzel. 1994. Neuromodulation, instruction and behavioral plasticity. In Flexibility and constraint in behavioral systems (ed. R. Greenspan and B. Kyriacou), pp. 109-118. J. Wiley \& Sons, Chichester, UK.

- - . 1995. Learning and memory in the honeybee. J. Neurosci. 15: 1617-1630.

Hammer, M., G. Braun, and J. Mauelshagen. 1994. Food induced arousal and nonassociative learning in honeybees: Dependence of sensitization on the application site and duration of food stimulation. Behav. Neural Biol. 62: 210-223.

Hasselmo, M.E., M.A. Wilson, B.P. Anderson, and J.M. Bower. 1990. Associative memory function in piriform (olfactory) cortex: Computational modeling and neuropharmacology. Cold Spring Harbor Symp. Quant. Biol.55: 599-610.

Heisenberg, M., A. Borst, S. Wagner, and D. Byers. 1985. D rosophila mushroom body mutants are deficient in olfactory learning. J. Neurogenetics 2: 1-30.

Hildebrandt, H. and U. M üller. 1995a. O ctopamine mediates rapid stimulation of protein kinase $A$ in the antennal lobe of honeybees. J. Neurobiol. 27: 44-50.

--1 . 1995b. PKA activity in the antennal lobe of honeybees is regulated by chemosensory stimulation in vivo. Brain Res. 679: 281-288.

Hodgson, T.M. and T. Crow. 1992. Cellular correlates of classical conditioning in identified light responsive pedal neurons of Hermissenda crassicornis. Brain Res. 570: $267-271$.

Kravitz, E.A. 1988. Hormonal control of behavior: Amines and the biasing of behavioral output in lobsters. Science 241: 1775-1781.

Kreissl, S., S. Eichmüller, G. Bicker, J. Rapus, and M. Eckert. 1994. O ctopamine-like immunoreactivity in the brain and suboesophageal ganglion of the honeybee. J. Comp. Neurol. 348: 583-595.

Laurent, G. and H. Davidowitz. 1994. Encoding of olfactory information with oscillating neural assemblies. Science 265: 1872-1875.

Laurent, G. and M. Naraghi. 1994. O dorant-induced oscillations in the mushroom bodies of the locust. J. Neurosci. 14: 2993-3004.

Linster, C. and B.H. Smith. 1997. A computational model of the response of honey bee antennal circuitry to odor 


\section{Hammer and Menzel}

mixtures: O vershadowing, blocking and unblocking can arise from lateral inhibition. Behav. Brain Res. 87: 1-14.

Malaka, R. and M. Hammer. 1996. Real-time models of classical conditioning. In Proceedings of the International Conference on Neural Networks (ICNN'96), Washington, pp. 768-773. IEEE Press, Piscataway, NJ.

Menzel, R. 1990. Learning, memory, and "cognition" in honey bees. In Neurobiology of comparative cognition (ed. R.P. Kesner and D.S. Olten), pp. 237-292. Erlbaum, Hillsdale, NJ.

Menzel, R. and U. Müller. 1996. Learning and memory in honeybees: From behavior to neural substrates. Annu. Rev. Neurosci. 19: 379-404.

Menzel, R., J. Erber, and T. Masuhr. 1974. Learning and memory in the honeybee. In Experimental analysis of insect behaviour (ed. L. Barton-Browne), pp. 195-217. Springer, Berlin, Germany.

Menzel, R., B. Michelsen, P. Rüffer, and M. Sugawa. 1988. Neuropharmacology of learning and memory in honey bees. In Synaptic transmission and plasticity in nervous systems (ed. G. Herting and H.C. Spatz), pp. 335-350. Springer, Berlin, Germany.

Menzel, R., S. Wittstock, and M. Sugawa. 1990. Chemical codes of learning and memory in the honey bee. In The biology of memory, Medica Hoechst Vol.23 (ed. L. Squire and K. Lindenlaub), pp. 335-360. Schattauer, Verlagsgesellschaft, Stuttgart, Germany.

Menzel, R., M. Hammer, G. Braun, J. Mauelshagen, and M. Sugawa. 1991. Neurobiology of learning and memory in honeybees. In The behaviour and physiology of bees (ed. L.J. Goodman and R.C. Fisher), pp. 323-353. CAB International, W allingford, UK.

M enzel, R., C. Durst, J. Erber, S. Eichmüller, M. Hammer, H. Hildebrandt, J. Mauelshagen, U. Müller, H. Rosenboom, J. Rybak, S. Schäfer, and A. Scheidler. 1994. The mushroom bodies in the honeybee: From molecules to behavior. In Neural basis of behavioral adaptations. Fortschritte der Zoologie Vol. 39 (ed. K. Schildberger and N. Elsner), pp. 81-102. Gustav Fischer Verlag, Stuttgart, Germany.

Mirenowicz, J. and W. Schultz. 1996. Preferential activation of midbrain dopamine neurons by appetitive rather than aversive stimuli. Nature 379: 449-451.

Mobbs, P.G. 1982. The brain of the honeybee Apis mellifera. I. The connections and spatial organization of the mushroom bodies. Phil. Trans. R. Soc. Lond. B 298: 309-354.

---.1984 . Neural networks in the mushroom bodies of the honeybee. J. Insect Physiol. 30: 43-58.

Rumelhart, D.E., G.E. Hinton, and R.J. Williams. 1986. Learning internal representations by error propagation. In Parallel distributed processing: Explorations in the microstructures of cognition (ed. D.E. Rumelhart and J.L. McClelland), pp. 318-362. MIT Press, Cambridge, MA.

Rybak, J. and R. Menzel. 1998. Integrative properties of the Pel-neuron, a unique Mushroom body output neuron. Learn. $\& M e m$. (this issue).

Schultz, W., P. Dayan, and P.R. Montague. 1997. A neural substrate of prediction and reward. Science 275: 1593-1599.

Skoulakis, M.C. and R.L. Davis. 1996. Olfactory learning deficits in mutants for leonardo, a Drosophila gene encoding a 14-3-3 protein. Neuron 17: 931-944.

Squire, L.R. 1987. Memory and brain. Oxford U niversity Press, New York, NY.

Stopfer, M., S. Bhagavan, B.H. Smith, and G. Laurent. 1997. Impaired odour discrimination on desynchronization of odour-encoding neural assemblies. Nature 390: 70-74.

Tully, T., T. Preat, S.C. Boynton, and M. Del Vecchio. 1994. Genetic dissection of consolidated memory in Drosophila. Cell 79: 35-47.

Received January 28, 1998; accepted in revised form April 14, 1998

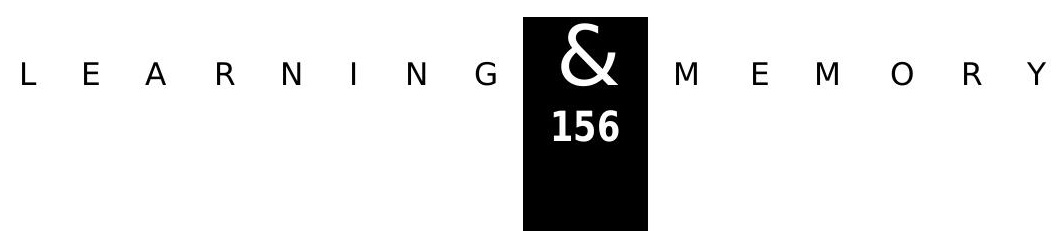




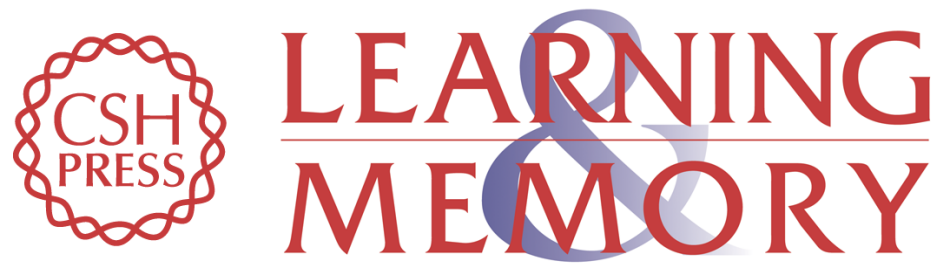

\section{Multiple Sites of Associative Odor Learning as Revealed by Local Brain Microinjections of Octopamine in Honeybees}

Martin Hammer and Randolf Menzel

Learn. Mem. 1998, 5:

Access the most recent version at doi:10.1101//m.5.1.146

References This article cites 32 articles, 8 of which can be accessed free at: http://learnmem.cshlp.org/content/5/1/146.full.html\#ref-list-1

License

Email Alerting Receive free email alerts when new articles cite this article - sign up in the box at the Service top right corner of the article or click here. 